\title{
STUDY OF THE OPTICAL ACTIVITY OF FERROELECTRIC LEAD GERMANATE
}

\author{
O.G. VLOKH, O.S. KUSHNIR AND Y.I. SHOPA \\ Physics Deptartment, Lviv State University \\ Lomonosowa st. 8, 290005 Lviv, Ukraine
}

(Received September 27, 1991; revised version February 7, 1992)

\begin{abstract}
A new optical method for measuring the optical activity of crystals is developed. Temperature dependence of gyration component $g_{11}$ of ferroelectric lead germanate is measured. The obtained results confirm that lead germanate may be considered as a gyroelectric crystal.
\end{abstract}

PACS numbers: $77.80 .-\mathrm{e}$

\section{In $\downarrow$ roduction}

Studying the optical activity (OA) of crystals for the light propagation directions different from the optical axes directions is connected with certain difficulties. Up to now few methods for this aim are developed, among them the methods of analysis of the light polarization characteristics $[1,2]$ and the methods basing on direct measurement of the light intensity [3, 4]. A new optical method for measurement of the OA of crystals is developed in this paper and successfully applied to investigation of $\mathrm{OA}$ of the ferroelectric lead germanate (LG) crystals $\left(\mathrm{Pb}_{5} \mathrm{Ge}_{3} \mathrm{O}_{11}\right)$. Investigations of temperature dependencies of LG OA had been performed earlier by several authors (see, for example [5-7]), but they had concerned mainly the optical axis direction (gyration component $g_{33}$ ). The temperature behaviour of $g_{11}$ component during ferroelectric phase transition was still unknown.

\section{Experimental method}

The present method for studying the $O A$ foresees the measurements of polarization azimuth $\chi$ and ellipticity $\varepsilon$ of the polarized light emergent from a crystal sample. The measurements of $\chi$ are performed by a "null" method in polarizer-sample-analyzer (PSA) optical system, and the measurements of $\varepsilon$ in polarizer-sample-compensator-analyzer (PSCA) system, where a quarter-wave plate is used as a compensator. Besides, the procedures of coordination of polarizer, 

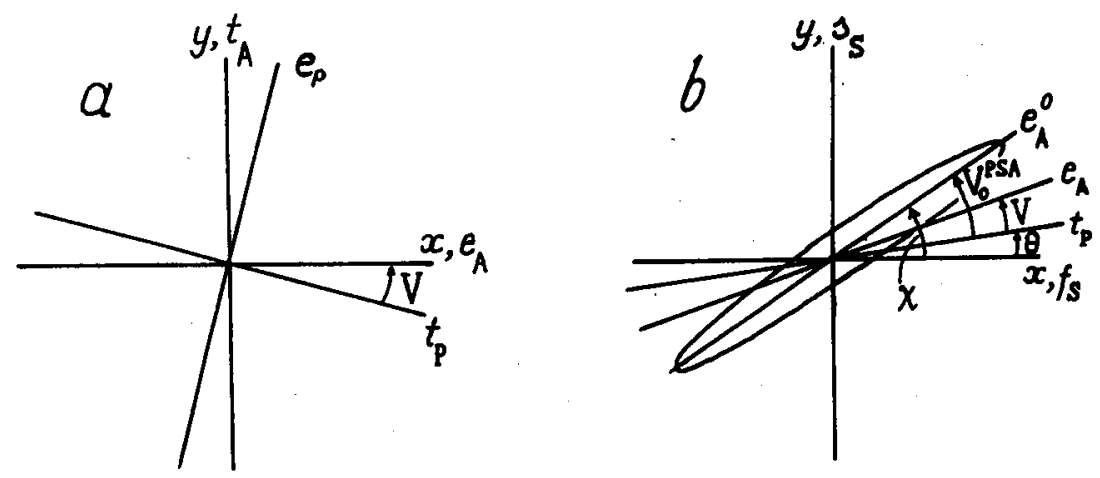

Fig. 1. Schematic orientation of optical elements in PA (a) and PSA (b) systems. $x, y$ are the axes of working frame of reference; $t_{\mathrm{P}}, e_{\mathrm{P}}-$ correspondingly the transmission and extinction axes of polarizer; $t_{\mathrm{A}}, e_{\mathrm{A}}$ - the same axes of analyzer; $f_{\mathrm{S}}, s_{\mathrm{S}}-$ the fast and slow axes of crystal sample. The analyzer axis position $e_{\mathrm{A}}^{0}$ corresponds to the minimum light intensity in PSA system.

analyzer and compensator scales in polarizer-analyzer (PA) and polarizer-compensator-analyzer (PCA) systems are also used. As it was done in $[3,4]$, polarization azimuths of the incident light (Fig. 1b) are chosen to be small. The values $\chi$ and $\varepsilon$ measured under such conditions are calculated [8] by Jones matrix method [9] (see formulae (A9), (A11) of Appendix). Experimental dependencies $\chi$ versus $\theta$ and $\varepsilon$ versus $\theta$ give the possibility to determine a characteristic ellipticity $\varepsilon_{0}$ related to an invariant polarization azimuth $\theta_{0}$ (see Appendix):

$$
\varepsilon_{0}=2 k-p_{0}+\delta \chi \cot (\Delta / 2)
$$

where $\Delta, k$ denote, respectively, the phase difference and the ellipticity of the eigenwaves in crystal. The effective parasitic parameters of the optical system, $p_{0}$ and $\delta \chi$, are defined by formulae (A15).

It is clear from Eqs. (A19), (A11) that a linear birefringence $\Delta n$ may be derived from the derivatives of $\chi$ versus $\theta$ and $\varepsilon$ versus $\theta$ lines. Using the analysis of experimental dependencies of $\varepsilon_{0}$ value on temperature on the basis of Eq. (1), the ellipticity $k$ and gyration $g=2 k \bar{n} \Delta n$ may be calculated, where $\bar{n}$ is the mean refractive index.

The suggested method unites the advantages of HAUP-method [4] (the use of small $\theta$ azimuths, were $\chi$ on $\theta$ and $\varepsilon$ on $\theta$ dependencies are linear, in contrast to [2], as well as the preventation of systematic errors due to consistent consideration of the imperfections of optical system) with the experimental simplicity of polarimetric azimuth measurements [1, 2] (accurate absolute intensity measurements $[3,4]$ require precise complex experimental apparatus). From analytical point of view the accuracies of measuring the OA and birefringence by HAUP-method and the present method must be same. Moreover as in [4], the present method enables to determine a rotation of optical indicatrix of crystals from the measurements of invariant azimuth $\theta_{0}$ position [8]. 


\section{Results and discussion}

The present method was applied to the measurement of the temperature dependence of $g_{11}$ component of LG (phase transition $\overline{6} \rightarrow 3$ at $T_{\mathrm{c}}=450 \mathrm{~K}$ ). The measurements were performed using a He-Ne laser beam (wavelength $632.8 \mathrm{~nm}$ ), which propagated along [100] direction of the specimen of $1.52 \mathrm{~mm}$ thickness. The temperature of specimen was controlled with the accuracy of $\pm 0.01 \mathrm{~K}$. The accuracy of azimuth measurements was about $5 \times 10^{-4} \mathrm{deg}$.

The procedure of determining the $\varepsilon_{0}$ value at $T=291.4 \mathrm{~K}$ is presented in Fig. 2 as an example. The measurements of absolute values of azimuths $\theta, \chi$

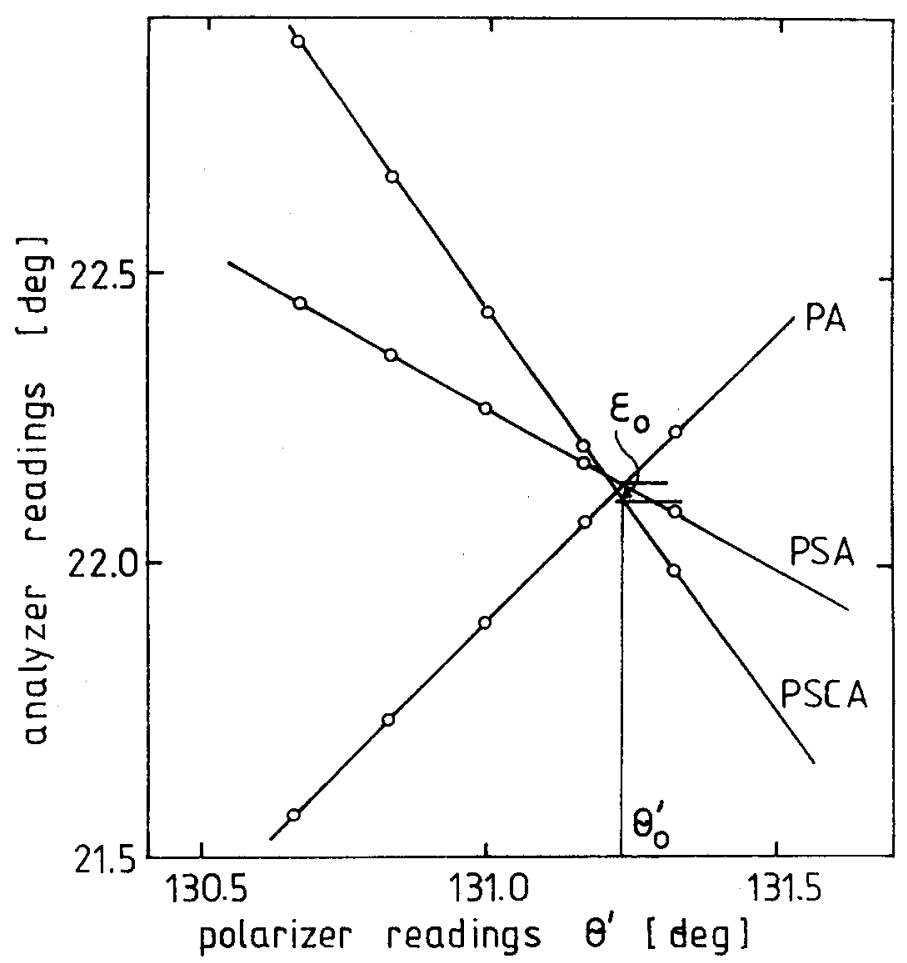

Fig. 2. Measurements of ellipticity $\varepsilon_{0}$ al $T=291.4 \mathrm{~K}$. The PSA and PSCA lines give $\chi^{\prime}$ and $\chi_{\mathrm{C}}^{\prime}$ values, correspondingly.

and $\chi_{\mathrm{C}}$ (see Appendix) are equivalent to the realization of accurate orientation of crystal sample in optical system. Since it is an extremely difficult problem [4], only relative values $\theta^{\prime}, \chi^{\prime}$ and $\chi_{C}^{\prime}$ are used in experiment. One can see that the showing of polarizer scale $\theta_{0}^{\prime}$, corresponding to the invariant azimuth $\theta_{0}$, may be determined from the intercept of PA and PSA lines (Fig. 2). Then, the ellipticity $\varepsilon_{0}$ is defined as $\varepsilon_{0}=\chi_{\mathrm{C}}^{\prime}-\chi^{\prime}$ at $\theta_{0}^{\prime}$ point. The value $\cos \Delta$ may be calculated simultaneously as

$$
\cos \Delta=\mathrm{d} \chi^{\prime} / \mathrm{d} \theta^{\prime}=\mathrm{d} \chi / \mathrm{d} \theta .
$$


The temperature dependencies of $\varepsilon_{0}$ and $\cos \Delta$ for single domain LG are depicted in Figs. 3a, 4a. Since both $p_{0}$ and $\delta \chi$ from Eq. (1) are assumed to be

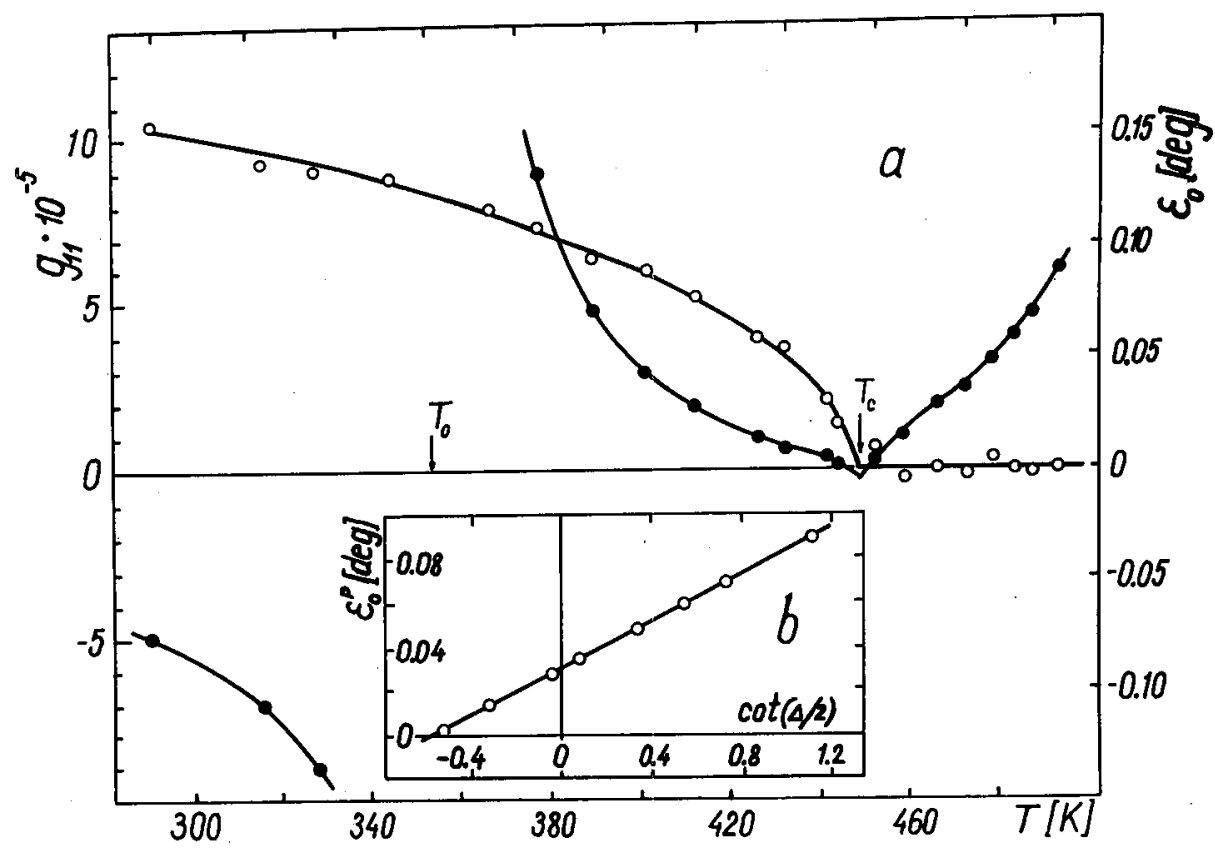

Fig. 3. Temperature dependencies of $\varepsilon_{0}(\bullet)$ and $g_{11}(\circ)$ for single domain LG (a) and $\varepsilon_{0}^{\mathrm{P}}$ on $\cot (\Delta / 2)$ dependence in paraelectric phase of LG (b).

independent of temperature, the dependence of $\varepsilon_{0}^{\mathrm{p}}$ on $\cot (\Delta / 2)$ for paraelectric phase, where $k=0$ according to symmetry considerations, must be linear. The experimental data are in good agreement with these assumptions (Fig. $3 \mathrm{~b}$ ). The values $p_{0}=-5.43 \times 10^{-4}$ and $\delta \chi=9.10 \times 10^{-4}$ are obtained on this basis. It enables to determine the temperature dependencies of $k$ and then $g_{11}$ (Fig. 3a). As it may be seen, the values $k, p_{0}$ and $\delta \chi$ are of the same order of magnitude, i.e. consideration of the polarizers imperfection parameters is a crucial condition of correct measurement of the OA of crystals.

The divergence of $\varepsilon_{0}$ at $T_{0}=353.9 \mathrm{~K}$ is only due to the temperature behaviour of the last term in Eq. (1). Really, since $\Delta=2 \pi m$ at $T_{0}$, where $m$ is integer (see also Fig. $4 \mathrm{a}$ ), the term $\delta \chi \cot (\Delta / 2)$ will have a divergence at this temperature.

The character of temperature dependence of $g_{11}$ component is similar to the one of $g_{33}$ component, the saturated values are about $9.9 \times 10^{-5}$ and $4.2 \times 10^{-5}$ $[6,7]$, respectively. The eroding of phase transition is insignificant. It must be noted that $g_{11}$ value obtained in the present paper differs essentially from the result for LG OA dispersion published earlier in [7] $\left(4.9 \times 10^{-5}\right.$ for $\left.633 \mathrm{~nm}\right)$. However, the experiments [7] were performed using a rough procedure of crystal sample orientation in optical system [1] and less precise apparatus. Moreover, the 


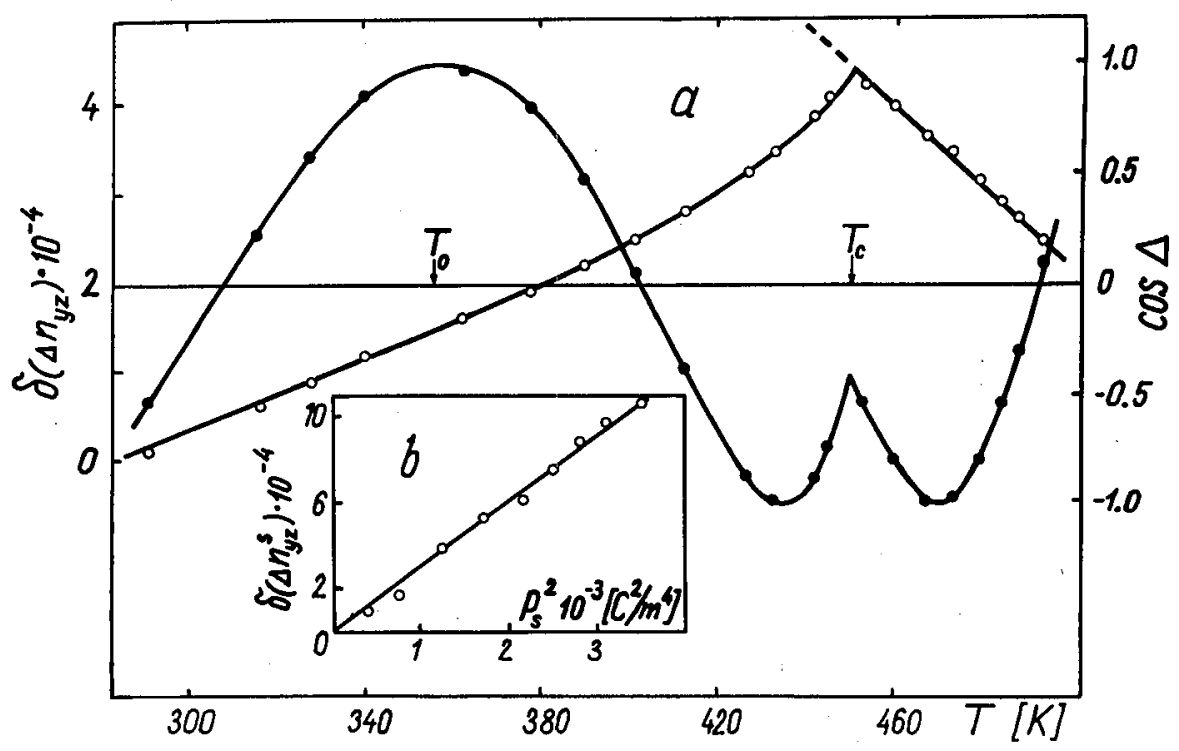

Fig. 4. Temperature dependencies of $\cos \Delta(\bullet)$ and $\delta\left(\Delta n_{y z}\right)(0)$ for LG (a) and relationship between the spontaneous increase $\delta\left(\Delta n_{y z}^{8}\right)$ and the square of spontaneous polarization $P_{\mathrm{s}}^{2}$ in ferroelectric phase of LG (b).

assumption about linear polarization of the incident light was used [1] with no consideration of the polarizers imperfections.

The ferroelectric LG may be considered as gyroelectric crystal, according to well-known Aizu classification [10], in spite of that the paraelectric phase of LG is not centrosymmetric. In fact, one can derive that $g_{11}$ depends linearly on spontaneous polarization $P_{\mathrm{s}}$ taken from [11] (Fig. 5):

$$
g_{11}=\gamma_{13}^{*} P_{\mathrm{s}} \text {. }
$$

Therefore, the OA of LG is explained completely by a linear spontaneous electrogyration effect, the corresponding coefficient being $\gamma_{13}^{*}=1.54 \times 10^{-3} \mathrm{~m}^{2} / \mathrm{C}$. Enantiomorphism of LG domains is confirmed also by the usual OA hysteresis loop obtained on repolarizing the specimen by external electric field (Fig. 6).

In general, the behaviour of the linear birefringence increment $\delta\left(\Delta n_{y z}\right)$ (Fig. 4b) obtained from the data of Fig. 4a is close to the one described in previous studies of single domain LG [7]. The spontaneous increase $\delta\left(\Delta n_{y z}^{\mathbf{s}}\right)$ in ferroelectric phase is well explained by a quadratic spontaneous electrooptic effect

$$
\delta\left(\Delta n_{y z}^{\mathrm{s}}\right)=\frac{1}{2}\left(n_{\mathrm{o}}^{3} M_{13}-n_{\mathrm{e}}^{3} M_{33}\right) P_{\mathrm{s}}^{2}=\frac{1}{2} M_{\mathrm{ef}} P_{\mathrm{s}}^{2},
$$

where $n_{\circ}$ and $n_{\mathrm{e}}$ are the ordinary and extraordinary refractive indices, respectively, $M_{13}, M_{33}$ and $M_{\mathrm{ef}}$ - the electrooptic coefficients. According to Fig. $4 \mathrm{~b}, M_{\mathrm{ef}}=$ $0.59 \mathrm{~m}^{4} / \mathrm{C}^{2}$. This does not differ strongly from the value $M_{\mathrm{ef}}=0.51 \mathrm{~m}^{4} / \mathrm{C}^{2}$ which may be calculated on the basis of the experimental data [7]. 

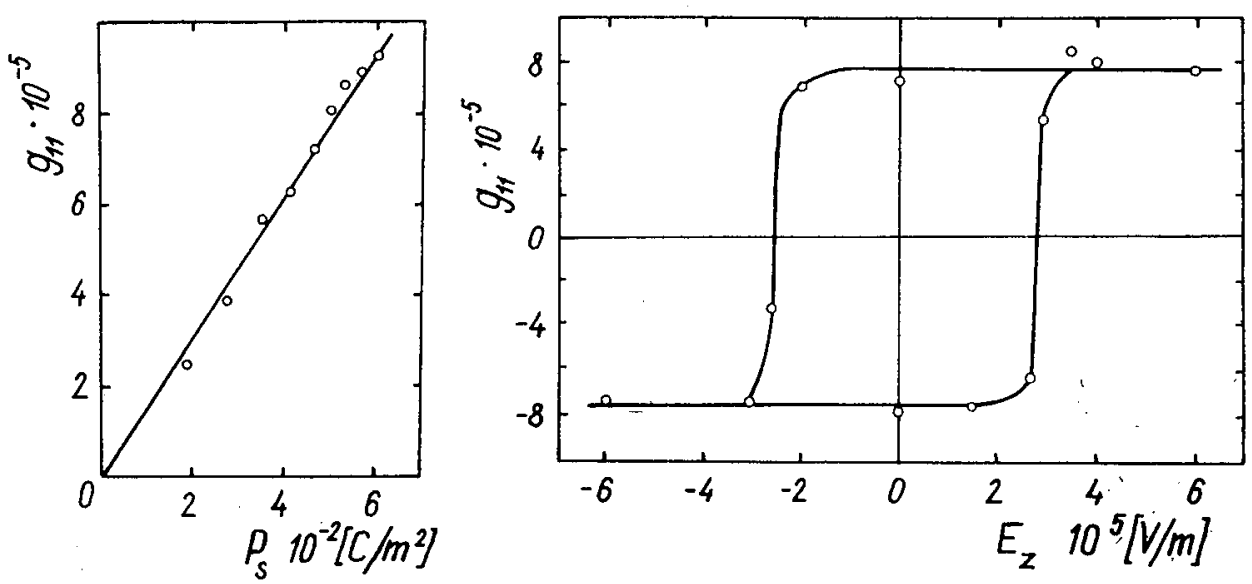

Fig. 5. Relationship between $g_{11}$ component and $P_{s}$ in ferroelectric phase of LG.

Fig. 6. Hysteresis loop of $g_{11}$ component displayed at $T=362.8 \mathrm{~K}$ for LG.

\section{Appendix}

The Jones vector $E_{\mathrm{A} 0}^{x y}$ of the light emergent from PA system* may be calculated [8] as

$$
\boldsymbol{E}_{\mathrm{A} 0}^{c y}=\boldsymbol{R}\left(-90^{\circ}\right) \boldsymbol{T}_{\mathrm{A}}^{t_{\mathrm{A}} e_{\mathrm{A}}} \boldsymbol{R}\left(90^{\circ}\right) \boldsymbol{R}(V) \boldsymbol{E}_{\mathrm{P} 0}^{t_{\mathrm{P}} e_{\mathrm{P}}}
$$

where $\boldsymbol{R}(V)$ is the matrix of rotation on $V$ angle (Fig. 1a), $\boldsymbol{E}_{\mathrm{PO}}^{t_{\mathrm{P}} \mathrm{P}_{\mathrm{P}}}-$ the Jones vector of the light emergent from polarizer

$$
\boldsymbol{E}_{\mathrm{p} 0}^{t_{\mathrm{p} e \mathrm{P}}}=\left(\begin{array}{c}
1 \\
\delta \theta+\mathrm{i} p
\end{array}\right)
$$

$\boldsymbol{T}_{\mathrm{A}}^{t_{\mathrm{A}} e_{\mathrm{A}}}$ - the Jones matrix of an ideal elliptic analyzer [9]:

$$
\boldsymbol{T}_{\mathrm{A}}^{t_{\mathrm{A}} e_{\mathrm{A}}}=\left(\begin{array}{cc}
1 & \delta V-\mathrm{i} q \\
\delta V+\mathrm{i} q & 0
\end{array}\right) .
$$

The parameters $p, q$ are the parasitic ellipticities of the light emergent from polarizer and analyzer, respectively, $\delta \theta$ and $\delta V$ - the corresponding angular imperfection parameters (see also [4]). Since the light intensity

$$
J=\boldsymbol{E}_{\mathrm{A} 0} \boldsymbol{E}_{\mathrm{A} 0}^{*}
$$

the value $V=V_{0}^{\mathrm{PA}}$ corresponding to minimum intensity in PA system with rotating analyzer may be obtained ${ }^{*}$ from the conditions $\left.(\mathrm{d} J / \mathrm{d} V)\right|_{V=V_{0}^{\mathrm{PA}}}=0$ and $\left.\left(\mathrm{d}^{2} J / \mathrm{d} V^{2}\right)\right|_{V=V_{0}^{\mathrm{PA}}}>0$ as

$$
V_{0}^{\mathrm{PA}}=\delta \theta_{\mathrm{PA}}-\delta V_{\mathbf{P A}} \text {. }
$$

*All the abbreviations coincide with the ones from [9], specifically the superscripts denote the axes defining the frame of reference in which Jones matrices and vectors are written.

*An approximation, linear on small values $p, q, \delta \theta, \delta V$ and others, is used in formulae for Jones matrices and vectors, and a quadratic one is used in formulae for light intensity. 
Since the conditions of incidence of light on polarizer are invariable for all discussed optical systems,

$$
\begin{aligned}
& p_{\mathrm{PA}}=p_{\mathrm{PCA}}=p_{\mathrm{PSA}}=p_{\mathrm{PSCA}}=p, \\
& \delta \theta_{\mathrm{PA}}=\delta \theta_{\mathrm{PCA}}=\delta \theta_{\mathrm{PSA}}=\delta \theta_{\mathrm{PSCA}}=\delta \theta .
\end{aligned}
$$

Therefore, the $\delta \theta$ parameter is accepted to be equal to zero due to redefinition of polarizer transmission axis position $[8,12]$. However, it is impossible to accept $\delta V$ to be equal to zero, because $\delta V$-parameter, depending on the conditions of incidence of light on analyzer, will change its value in various optical systems $[13]$, i.e. $\delta V_{\mathrm{PA}} \neq \delta V_{\mathrm{PCA}}$, etc. The analyzer parameter $q$ also will be changeable $\left(q_{\mathrm{PA}} \neq q_{\mathrm{PCA}}\right.$, etc. $)$.

Under the condition of small azimuths $\theta$ of incident light the Jones vector of the light emergent from analyzer in PSA system will be (Fig. 1b):

$$
\boldsymbol{E}_{\mathrm{A} 0}^{x y}=\boldsymbol{R}\left(-90^{\circ}-\theta-V\right) \boldsymbol{T}_{\mathrm{A}}^{t_{\mathrm{A}} e_{\mathrm{A}}} \boldsymbol{R}\left(-90^{\circ}+\theta+V\right) \boldsymbol{T}_{\mathrm{S}}^{x y} \boldsymbol{R}(-\theta) \boldsymbol{E}_{\mathrm{P} 0}^{t_{\mathrm{P}} e_{\mathrm{P}}},
$$

where $T_{\mathrm{S}}^{x y}$ is the Jones matrix of gyrotropic birefringent crystal for the case of light propagation directions essentially different from the optical axes directions $(k \ll 1)$ :

$$
T_{\mathrm{S}}^{x y}=\left(\begin{array}{cc}
\exp (-\mathrm{i} \Delta / 2) & -2 k \sin (\Delta / 2) \\
2 k \sin (\Delta / 2) & \exp (\mathrm{i} \Delta / 2)
\end{array}\right) .
$$

Note that the abbreviations $q=q_{\mathrm{PSA}}$ and $\delta V=\delta V_{\mathrm{PSA}}$ are introduced into Eqs. (A3), (A7). The value $V=V_{0}^{\mathrm{PSA}}$ corresponding to the minimum of $J$ on $V$ dependence may be calculated, and the polarization azimuth $\chi$ of the light emergent from a crystal sample may be obtained on this basis as

$$
\chi=\theta \cos \Delta+(k-p) \sin \Delta-\delta V_{\mathrm{PSA}},
$$

since $V_{0}^{\mathrm{PSA}}=\chi-\theta$ (Fig. 1b).

The ellipticity $\varepsilon$ of the light passing through a crystal sample may be experimentally found from measurements, with the use of minimum intensity method in PSCA system, of the polarization azimuth $\chi_{\mathrm{C}}$ of the light emergent from a quarter-wave plate under the condition that the principal axis of a quarter-wave plate coincides with the position $e_{\mathrm{A}}^{0}$ of analyzer axis in PSA system (Fig. 1b):

$$
\varepsilon=\chi_{\mathrm{C}}-\chi \text {. }
$$

The last condition may be realized using the results of coordinating the polarizer, compensator and analyzer scales in PCA system.

The relationship for $\varepsilon$ may be obtained after applying Jones matrix method in a similar manner to the analysis of PCA and PSCA systems [8]:

$$
\begin{aligned}
& \varepsilon=\theta \sin \Delta+k(1-\cos \Delta)+p \cos \Delta \\
& +\frac{1}{2}\left(-p-q_{\mathrm{PCA}}+\delta V_{\mathrm{PA}}+\delta V_{\mathrm{PCA}}-2 \delta V_{\mathrm{PSCA}}\right) .
\end{aligned}
$$

Since it is difficult to measure absolute values of $\theta$ and $\chi$ azimuths (see, for example [4]), we must register an equality of the azimuths of the incident light and the light emergent from a sample (so-called invariant azimuth) on the basis of measurements in PA system, i.e. the invariant azimuth $\theta_{0}$ will be defined only with the accuracy of $\delta V_{\mathrm{PA}}$ :

$$
\chi_{0}=\theta_{0}-\delta V_{\mathrm{PA}} \text {. }
$$


Inserting Eq. (A12) into (A9) and (A11), we shall obtain $\theta_{0}$ value

$$
\theta_{0}=(k-p) \cot (\Delta / 2)+\delta \chi / 2 \sin ^{2}(\Delta / 2)
$$

and then the value of the characteristic ellipticity $\varepsilon_{0}=\varepsilon\left(\theta_{0}\right)[8]$ :

$$
\varepsilon_{0}=2 k-p_{0}+\delta \chi \cot (\Delta / 2)
$$

where

$$
\begin{aligned}
& p_{0}=\frac{1}{2}\left(3 p+q_{\mathrm{PCA}}-\delta V_{\mathrm{PA}}-\delta V_{\mathrm{PCA}}+2 \delta V_{\mathrm{PSCA}}\right), \\
& \delta \chi=\delta V_{\mathrm{PA}}-\delta V_{\mathrm{PSA}} .
\end{aligned}
$$

It is clear from Eqs. (A14), (A15) that the influence of angular parameter $\delta V$ on $\varepsilon_{0}$ value vanishes under the ideal condition of equality of $\delta V$ values for all optical systems in which $\chi$ and $\varepsilon$ are measured.

\section{References}

[1] A.F. Konstantinova, N.R. Ivanov, B.N. Grechushnikov, Kristallografiya 14, 283 (1969).

[2] A.I. Okorochkov, A.F. Konstantinova, L.V. Soboleva, L.I. Hapayeva, Kristallografiya 29, 1102 (1984).

[3] J. Kobayashi, T. Takahashi, T. Hosokawa, Y. Uesu, J. Appl. Phys. 49, 809 (1978).

[4] J. Kobayashi, Y. Uesu, J. Appl. Cryst. 16, 204 (1983); J. Kobayashi, H. Kumomi, K. Saito, J. Appl. Cryst. 19, 377 (1986).

[5] H. Iwasaki, K. Sugii, Appl. Phys. Lett. 19, 92 (1971).

[6] O.G. Vlokh, L.A. Lazko, Y.I. Shopa, J. Phys. Soc. Jpn., Suppl. B 49, 150 (1980).

[7] O.G. Vlokh, L.A. Lazko, Y.I. Shopa, Phys. Status Solidi A 65, 371 (1981).

[8] O.G. Vlokh, O.S. Kushnir, Y.I. Shopa, I.V. Bereznoy, Preprint No. 6-91 of the Inst. of Appl. Problems of Mechanics and Mathematics, Acad. Sci. of Ukraine, Lviv 1991, p. 22.

[9] R. Azzam, N. Bashara, Ellipsometry and Polarized Light, Mir, Moscow 1981, p. 584.

[10] K. Aizu, Phys. Rev. A 133, 1584 (1964).

[11] O.G. Vlokh, E.V. Sinyakov, L.A. Lazko, Y.I. Shopa, A.Y. Kreycherek, Fiz. Tverd. Tela 22, 227 (1980).

[12] D.E. Aspnes, J. Opt. Soc. Am. 61, 1077 (1971).

[13] J. Kobayashi, T. Asahi, S. Takahashi, A.M. Glazer, J.Appl. Cryst. 21, 479 (1988). 